BULLETIN OF THE

AMERICAN MATHEMATICAL SOCIETY

Volume 80, Number 1, January 1974

\title{
RESTRICTED IDEALS IN RINGS OF ANALYTIC FUNCTIONS
}

\author{
BY ANDREW ADLER AND R. DOUGLAS WILLIAMS
}

Communicated by Creighton Buck, May 16, 1973

Introduction. Let $Y$ be a connected, noncompact Riemann surface, and let $A$ be the ring of all analytic functions on $Y$. It is known that the ideal theory of the ring $A$ is strikingly similar to the ideal theory of the ring $C(X)$ of all real valued continuous functions on a completely regular topological space $X$. We show that locally much of the ideal theory of $A$ can be recovered from the ideal theory of $C(\Sigma)$ for a particular space $\Sigma$. This will provide a device for transforming results about the ideal theory of $C(\Sigma)$ into results about the ideal theory of $A$.

1. Let $M$ be a free maximal ideal of $A$, and let $P^{*}$ denote the ideal $\bigcap_{n \in N} M^{n} . P^{*}$ is the largest prime ideal properly contained in $M$. Let $A_{P^{*}}$ be the localization of $A$ at $P^{*}$. We show in this section that the ideal theory of $A_{P^{*}}$ is essentially the same as the ideal theory of $C(\Sigma) / P$ for a suitably chosen space $\Sigma$ and a suitably chosen minimal prime ideal $P$ of $C(\Sigma)$. Let $t \in M-\{0\} . Z(t)$, the set of zeros of $t$, is a countably infinite closed discrete subset of $Y$. Denote $Z(t)$ by $N$; we think of $Z(t)$ as a copy of the space $N$ of positive integers. The collection

$$
\mu=\{Z(f) \cap N: f \in M\}
$$

is a free ultrafilter on $N$ and hence corresponds to a point $\sigma$ of $\beta N-N$. Let $\Sigma$ be the space $N \cup\{\sigma\}$, where $\Sigma$ has the relative topology of $\beta N$, and let $P$ be the minimal prime ideal of $C(\Sigma)$ given by

$$
P=\{f \in C(\Sigma): Z(f) \cap N \in \mu\} .
$$

The ideals of $A_{P^{*}}$ (respectively $C(\Sigma) / P$ ) under multiplication of ideals and inclusion form an ordered semigroup $\mathscr{I}\left(A_{P^{*}}\right)$ (respectively $\mathscr{I}(C(\Sigma) / P))$.

Proposition 1. There exists an order preserving isomorphism of $\mathscr{I}\left(A_{P^{*}}\right)$ onto $\mathscr{I}(C(\Sigma) / P)$ that maps the set of principal ideals of $A_{P^{*}}$ onto the set of principal ideals of $C(\Sigma) / P$.

AMS (MOS) subject classifications (1970). Primary 46E25; Secondary 13A15.

Key words and phrases. Rings of analytic functions, rings of continuous functions, ideals, valuation rings, ultrapowers. 
OUtLINE OF PROOF. The ring $A_{P^{*}}$ is a valuation ring [1]. We describe its value group. Let $Z$ be the integers considered as an ordered additive group. Form the ultrapower $Z^{N} / \mu$ [2]. Identify elements of $Z^{N} / \mu$ that differ by a standard integer. The quotient group thus obtained is the value group of $A_{P^{*}} . C(\Sigma) / P$ is also a valuation ring [4, Theorem 14.24]. The value group of $C(\Sigma) / P$ is a quotient $G / H$ of two multiplicative groups. $G$ is the multiplicative group of the field $R^{N} / \mu$, where $R$ denotes the reals. $H$ is the group of noninfinitesimal bounded elements of $G$. This value group is ordered as follows: $[f / \mu] \geqq 0$ if, and only if, $f / \mu$ is bounded.

The statement of Proposition 1 is equivalent to the statement that there exists an order preserving isomorphism of these two value groups. Now one can verify that the map given by

$$
[f / \mu] \mapsto[g / \mu], \quad \text { where } g(n)=\exp (-f(n)),
$$

is an order preserving isomorphism of the first value group onto the second.

From Proposition 1 it follows that any proposition of ideal theory that involves only multiplication of ideals, inclusion, and principalness is true for $C(\Sigma) / P$ if, and only if, it is true for $A_{P^{*}}$.

If $M_{1}$ and $M_{2}$ are distinct free maximal ideals of $A$, it is natural to ask to what degree the associated rings $A_{P_{1}^{*}}$ and $A_{P_{2}^{*}}$ differ. From a deep result of Iss'sa [6] it follows that these rings need not be isomorphic (see [8, p. 299]). If we assume the continuum hypothesis, however, all ultrapowers of $Z$ using countable index set and free ultrafilters are isomorphic [7]. It follows that $A_{P_{1}^{*}}$ and $A_{P_{2}^{*}}$ have the same value group and therefore the same ideal theory in the sense of Proposition 1.

2. In this section we consider the restricted ideals of $A$ that are contained in $P^{*}$, i.e. the ideals of the form $I \cap A$, where $I$ is an ideal of $A_{P^{* *}}$. We call such ideals $P^{*}$-restricted ideals. This class of ideals properly includes all nonmaximal prime ideals of $A$ that are contained in $M$ and all the primary ideals of $A$ that are contained in $M$, except for the powers $M^{n}$ of $M$. We show that the $P^{*}$-restricted ideals of $A$ behave essentially like the ideals of $A_{P^{*}}$, and hence by Proposition 1 essentially like the ideals of $C(\Sigma) / P$.

Definitions. (1) Let $I$ be an ideal of $A$. Set

$$
I_{*}=\left\{f \in A: f h \in I \text { for some } h \in A-P^{*}\right\} .
$$

(2) If $J=(g)_{*}$ for some $g \in A$, we say that $J$ is $P^{*}$-principal.

Using the generalized Weierstrass product theorem [3] one can show that the set of $P^{*}$-restricted ideals is closed under multiplication and $I_{*} J_{*}=(I J)_{*}$ for any ideals $I$ and $J$ of $A$. Therefore we have 
Proposition 2. The map $I \mapsto I \cap A$ is an order preserving isomorphism of $\mathscr{I}\left(A_{P^{*}}\right)$ onto the multiplicative semigroup of $P^{*}$-restricted ideals of $A$. This isomorphism carries the set of principal ideals of $A_{P^{*}}$ onto the set of $P^{*}$-principal ideals of $A$.

Let $\mathscr{I}(A)$ be the multiplicative semigroup of ideals of $A$. Note that if $I \in \mathscr{I}(A)$ and $J$ is a $P^{*}$-restricted ideal, then $I \subset J$ if, and only if, $I_{*} \subset J$. Combining this observation with Propositions 1 and 2 we have

THEOREM. There is a map $\phi$ from $\mathscr{I}(A)$ onto $\mathscr{I}(C(\Sigma) / P)$ with the following properties:

(1) $\phi$ is a semigroup homomorphism.

(2) The restriction of $\phi$ to the $P^{*}$-restricted ideals of $A$ is a surjective order preserving isomorphism.

(3) $\phi$ takes principal (and $P^{*}$-principal) ideals of $A$ to principal ideals of $C(\Sigma) / P$.

(4) If $I \in \mathscr{I}(A)$ and $J$ is a $P^{*}$-restricted ideal, then $I \subset J$ if, and only if, $\phi(I) \subset \phi(J)$.

The theorem gives rise to a useful transfer principle, which we state semiformally. We will need some terminology. Let $f_{1}, f_{2}, \cdots ; I_{1}, I_{2}, \cdots$; $J_{1}, J_{2}, \cdots ; n_{1}, n_{2}, \cdots$ be first order variable letters. The $n_{i}$ will range over the natural numbers. Any expression of the form $\left(f_{i}\right), I_{i}$, or $J_{i}$ will be a term. If $S, T$ are terms, let $S T$ and $S^{n_{i}}$ also be terms. An atomic firstorder formula will be an expression of the form $S \subset T$, where $S, T$ are terms and $T$ contains no occurrences of any $\left(f_{i}\right)$ or $J_{i}$. Now let $\mathscr{S}$ be a sentence of higher-order logic (see e.g. [10] for precise definitions) whose first-order components are built up from our atomic first-order formulas. We have then

TRANSFER PRINCIPLE. $\mathscr{S}$ is true in $A$, where the $f_{i}$ range over elements of $A$, the $J_{i}$ range over ideals of $A$, and the $I_{i}$ range over $P^{*}$-restricted ideals of $A$, if, and only if, $\mathscr{S}$ is true in $C(\Sigma) / P$, where the $f_{i}$ range over elements of $C(\Sigma) / P$, and the $J_{i}$ and $I_{i}$ range over ideals of $C(\Sigma) / P$.

3. In this section we present some results of ideal theory that hold in $C(\Sigma) / P$ and hence by the transfer principle (or the theorem) hold automatically for the $P^{*}$-restricted ideals of $A$. These results (except for possibly Examples 5(b) and 5(c)) are known for both rings but have been proved by techniques which appear on the surface to be quite different.

EXAMPLE 1. $I$ is prime if, and only if, $I=I^{2}$. This sentence can be written as follows:

$$
\mathscr{S}: \forall I\left\{I \subset I^{2} \wedge I^{2} \subset I \leftrightarrow \forall f \forall g[(f)(g) \subset I \rightarrow((f) \subset I \vee(g) \subset I)]\right\}
$$


This sentence clearly has the form described in the statement of the transfer principle. (It is actually a sentence of first-order logic.) $\mathscr{S}$ is true in $C(X) / P$ for all $X$ and all prime ideals $P$ of $C(X)$ [11, Corollary 2.2]. Hence for any $P^{*}, \mathscr{S}$ is true for the $P^{*}$-restricted ideals of $A$. This is proved directly in [12].

For the remaining examples we omit the verification that the sentences can be expressed in the form required by the transfer principle.

EXAMPLE 2. $I$ is primary if, and only if, either

$$
I=I \cdot I^{1 / 2} \quad \text { or } \quad I=I: I^{1 / 2} .
$$

This is true in $C(\Sigma) / P$ by [11, Corollary 2.10]. It is therefore true for the $P^{*}$-restricted ideals of $A$ (proved directly in [12]).

EXAMPLE 3. Every nonprime primary ideal is either an upper or a lower primary ideal. This is proved for $C(X) / P$ for arbitrary $X$ in [9]. Note that the transfer principle enables one to avoid some fairly complex machinery necessary to prove this result for the $P^{*}$-restricted ideals of $A$ (see [12, Theorem 2.2]).

EXAMPLE 4. The set of all upper prime ideals properly between two given ones is an $\eta_{1}$-set. For $C(X) / P$ this is [4, Theorem 14.9(b)]. The result for the $P^{*}$-restricted ideals of $A$ is essentially contained in [5].

EXAMPLE 5. (a) No nonzero prime ideal is finitely generated. (b) Every upper prime ideal is generated by a countable family of $P^{*}$-principal ideals. (c) No lower prime ideal is countably generated. For $C(X) / P$ see $[4,14 C]$.

\section{REFERENCES}

1. N. L. Alling, The valuation theory of meromorphic function fields over open Riemann surfaces, Acta Math. 110 (1962), 79-96. MR 28 \#3992.

2. J. L. Bell and A. B. Slomson, Models and ultraproducts: An introduction, NorthHolland, Amsterdam, 1969. MR 42 \#4381.

3. H. Florack, Reguläre und meromorphe Funktionen auf nicht geschlossenen Riemannschen Flächen, Schr. Math. Inst. Univ. Münster, no. 1 (1948). MR 12, 251.

4. L. Gillman and M. Jerison, Rings of continuous functions, University Series in Higher Math., Van Nostrand, Princeton, N.J., 1960. MR 22 \#6994.

5. M. Henriksen, On the prime ideals of the ring of entire functions, Pacific J. Math. 3 (1953), 711-720. MR 15, 537.

6. H. Iss'sa, On the meromorphic function field of a Stein variety, Ann. of Math. (2) 83 (1966), 34-46. MR 32 \#2613.

7. H. J. Keisler, A survey of ultraproducts, Logic Methodology and Philos. Sci. (Proc, 1964 Internat. Congr.), North-Holland, Amsterdam, 1965, pp. 112-126. MR 34 \#5678.

8. J. Kelleher, Rings of meromorphic functions on non-compact Riemann surfaces, Canad. J. Math. 21 (1969), 284-300. MR 39 \#1670.

9. C. W. Kohls, Primary ideals in rings of continuous functions, Amer. Math. Monthly 71 (1964), 980-984. MR 30 \#2332. 
10. R. Montague, Set theory and higher order logic, Formal Systems and Recursive Functions (Proc. Eighth Logic Colloq., Oxford, 1963), North-Holland, Amsterdam, 1965, pp. 131-148. MR 36 \#3638.

11. R. D. Williams, Intersections of primary ideals in rings of continuous functions, Canad. J. Math. 24 (1972), 502-519. MR 45 \#4134.

12. - Primary ideals in rings of analytic functions, Trans. Amer. Math. Soc. 177 (1973), 37-49.

Department of Mathematics, University of British Columbia, Vancouver 8, British Columbia, CaNada 